\title{
Mean-Square Approximation of Complex Variable Functions by Fourier Series in the Weighted Bergman Space
}

\author{
M.Sh. Shabozov ${ }^{1, a}$ and M.S. Saidusaynov ${ }^{2, b}$ \\ ${ }^{1}$ A.Juraev Institute of Mathematics, Academy of Science of the Republic of Tajikistan, \\ Ayni str., 299/4, Dushanbe, Tajikistan, 734063 \\ Russian-Tajik Slavonic University, M.Tursunzoda str., 30, Dushanbe, Tajikistan, 734000 \\ ${ }^{2}$ Tajik National University, Rudaky ave., 17, Dushanbe, Tajikistan, 734025 \\ ashabozov@mail.ru, bsmuqim@gmail.com
}

Keywords: weighted Bergman space, generalized module of continuity, generalized displacement operator.

\begin{abstract}
The problem of mean-square approximation of complex variables functions regularly in some simply connected domain $\mathcal{D} \subset \mathbb{C}$ with Fourier series by orthogonal system when the weighted function $\gamma:=\gamma(|z|)$ is nonnegative integrable in $\mathcal{D}$, was considered. An exact convergence rate of Fourier series by orthogonal system of functions on some class of functions given by special module of continuity of $m$-th order were obtained. An exact values of $n$-widths for specified class of functions were calculated.
\end{abstract}

\section{Introduction and Preliminary Results}

In this paper, the quadratic approximation of functions with Fourier series by orthogonal system over complex variable domain in the presence of weight was considered.

In the domain of $\mathcal{D} \subset \mathbb{C}$ is given a nonnegative measurable and not equivalent zero function $\gamma(|z|)$, such that there is exists a finite integral

$$
\iint_{(\mathcal{D})} \gamma(|z|) d \sigma>0
$$

where the integral is understood in sense of Lebesgue and $d \sigma$ the element of area. Function $\gamma=\gamma(|z|)$ satisfying the above condition we call a weight function.

We will consider the problems of mean square approximation by Fourier sum of complex function $f$ regularly in the simply connected domain $\mathcal{D}$ and is belong to the space $L_{2, \gamma}:=L_{2}(\gamma(|z|), \mathcal{D})$ with finite norm

$$
\|f\|_{2, \gamma}:=\|f\|_{L_{2, \gamma}}=\left(\iint_{(\mathcal{D})} \gamma(|z|)|f(z)|^{2} d \sigma\right)^{1 / 2}<\infty,
$$

where $\gamma(|z|)$ weighted function in the domain $\mathcal{D}$. Where the domain $\mathcal{D}$ is disk $|z|<R$ $(0<R<\infty)$ the $L_{2, \gamma}$ space is a Bergman space $B_{2, \gamma}$ introduced in $[1,2]$. An extremal problems of analytic functions and the problem of calculation of different values of $n$-widths in the space $B_{2, \gamma}$ are considered in works (see, e.g., [3-7]).

The results which are obtained in this paper are the generalization and continuation of work [9]. We shall indicate some other papers that are close to our work in which the analogy questions for other orthogonal system of functions [10-13] were studied, and therefore, we bring the necessary definitions and facts from it for further studying.

Let $\left\{\varphi_{k}(z)\right\}_{k=0}^{\infty}$ be complete orthonormal system in domain $\mathcal{D}$ of a system of complex functions in the space $L_{2, \gamma}$ :

$$
f(z)=\sum_{k=0}^{\infty} c_{k}(f) \varphi_{k}(z), \quad c_{k}(f)=\iint_{(\mathcal{D})} \gamma(|z|) f(z) \overline{\varphi_{k}(z)} d z
$$


are the Fourier series of function $f \in L_{2, \gamma}$ under this system,

$$
S_{n}(f ; z)=\sum_{k=0}^{n-1} c_{k}(f) \varphi_{k}(z)
$$

are the partial sums of $n$ order. Let $\mathcal{P}_{n}$ be the subspace of generalized complex polynomials of form

$$
p_{n}(z)=\sum_{k=0}^{n-1} d_{k} \varphi_{k}(z)
$$

where $d_{k} \in \mathbb{C}$. Then, as it is well known (see, e.g., [8], p.263):

$$
\begin{gathered}
E_{n-1}(f)=\inf \left\{\left\|f-p_{n}\right\|_{2, \gamma}^{2}: p_{n}(z) \in \mathcal{P}_{n}\right\}= \\
=\left\|f-S_{n}(f)\right\|_{2, \gamma}^{2}=\sum_{k=n}^{\infty}\left|c_{k}(f)\right|^{2},
\end{gathered}
$$

where $c_{k}(f)$ are the Fourier coefficients of function $f$ defined in (1).

Now consider the function

$$
T(\xi, \eta ; h)=\sum_{k=0}^{\infty} \varphi_{k}(\xi) \overline{\varphi_{k}(\eta)} h^{k},
$$

where $h \in(0,1),(\xi, \eta) \in \mathcal{D} \times \mathcal{D}$, and the series in the right side of (3) is understood in the meaning of convergence in the space $L_{2}(\mathcal{D} \times \mathcal{D} ; \gamma(|\xi|) \gamma(|\eta|))$. Just note that in some cases we can show the explicit form for the function $T(\xi, \eta ; h)$. Thus, for example, if $\mathcal{D}=\{z \in \mathbb{C} ;|z|<1\}, \gamma(|z|)=1$, then the system of functions $\varphi_{k}(z)=\sqrt{(k+1) / \pi} z^{k}, k=0,1, \ldots$ is orthonormalized (see, e.g., [8, p.208]). In this case, we have (see, e.g., [9]):

$$
\begin{gathered}
T(\xi, \eta ; h)=\sum_{k=0}^{\infty} \varphi_{k}(\xi) \overline{\varphi_{k}(\eta)} h^{k}= \\
=\frac{1}{\pi} \sum_{k=0}^{\infty}(k+1)(\xi \eta h)^{k}=\frac{1}{\pi} \cdot \frac{1}{(1-\xi \bar{\eta} h)^{2}} .
\end{gathered}
$$

We return to the general case of the domain $\mathcal{D}$. In $L_{2, \gamma}$ space, we shall consider an operator

$$
F_{h} f(z)=\iint_{(\mathcal{D})} \gamma(|\zeta|) f(\zeta) T(z, \zeta ; 1-h) d \zeta,
$$

which is called generalized translation operator. The operator $F_{h}(f)$ has the following properties:

1) $F_{h}\left(f_{1}+f_{2}\right)=F_{h}\left(f_{1}\right)+F_{h}\left(f_{2}\right), \quad$ 2) $F_{h}(\lambda f)=\lambda F_{h}(f), \lambda \in \mathbb{C}$

3) $\left\|F_{h}(f)\right\| \leq\|f\|$, 4) $F_{h} \varphi_{k}(z)=(1-h)^{k} \varphi_{k}(z)$,

5) $\left\|F_{h}(f)-f\right\| \rightarrow 0, \quad h \rightarrow 0$.

Using the generalized translation operator $F_{h}(f)$ for an arbitrary function $f \in L_{2, \gamma}$, we define the finite-difference of first and higher order by the equations

$$
\begin{gathered}
\Delta_{h}^{1} f(z)=f(z)-F_{h} f(z)=\left(\mathbb{I}-F_{h}\right) f(z), \\
\Delta_{h}^{m} f(z)=\Delta_{h}\left(\Delta_{h}^{m-1} f(z)\right)=\left(\mathbb{I}-F_{h}\right)^{m} f(z)= \\
=\sum_{k=0}^{m}(-1)^{m-k}\left(\begin{array}{l}
m \\
k
\end{array}\right) F_{h}^{(k)} f(z),
\end{gathered}
$$


where $F_{h}^{0} f(z)=\mathbb{I} f(z)=f(z), F_{h}^{(k)} f(z)=F_{h}\left(F_{h}^{(k-1)} f(z)\right), k=\overline{1, m}, m \in \mathbb{N}, \mathbb{I}-$ unit operator in the space $L_{2, \gamma}$. The magnitude

$$
\Omega_{m}(f ; t)_{2, \gamma}=\sup \left\{\left\|\Delta_{h}^{m} f\right\|_{2, \gamma}: 0<h \leq t\right\}
$$

we call a generalized module of continuity of $m$-th order of function $f \in L_{2, \gamma}$.

Further, we need the following simple lemma.

Lemma 1. For an arbitrary function $f \in L_{2, \gamma}$ is hold

$$
\Omega_{m}^{2}(f ; t)_{2, \gamma}=\sum_{k=0}^{\infty}\left(1-(1-t)^{k}\right)^{2 m}\left|c_{k}(f)\right|^{2} .
$$

Proof. First, it is observed that an operator (4) with respect of (3) is representable in form

$$
\begin{gathered}
F_{h} f(z)=\iint_{(\mathcal{D})} \gamma(|\zeta|) f(\zeta) T(z, \zeta ; 1-h) d \zeta= \\
=\iint_{(\mathcal{D})} \gamma(|\zeta|) f(\zeta)\left\{\sum_{k=0}^{\infty} \varphi_{k}(z) \overline{\varphi_{k}(\zeta)}(1-h)^{k}\right\} d \zeta= \\
=\sum_{k=0}^{\infty}\left(\iint_{(\mathcal{D})} \gamma(|\zeta|) f(\zeta) \overline{\varphi_{k}(\zeta)} d \zeta\right) \varphi_{k}(z)(1-h)^{k}= \\
=\sum_{k=0}^{\infty} c_{k}(f) \varphi_{k}(z)(1-h)^{k},
\end{gathered}
$$

using which we find consecutively

$$
\Delta_{h} f(z):=f(z)-F_{h} f(z)=\sum_{k=0}^{\infty} c_{k}(f) \varphi_{k}(z)\left(1-(1-h)^{k}\right) .
$$

Then using the obtained formula for any $m \in \mathbb{N}$ and $h \in(0,1)$ we find:

$$
\Delta_{h}^{m} f(z)=\Delta\left(\Delta_{h}^{m-1} f(z)\right)=\sum_{k=0}^{\infty} c_{k}(f) \varphi_{k}(z)\left(1-(1-h)^{k}\right)^{m}
$$

Applying the Parseval equality for (7) and because of system of functions $\left\{\varphi_{k}(z)\right\}_{k=0}^{\infty}$ are orthonormal in the domain of $\mathcal{D} \subset \mathbb{C}$ we write

$$
\left\|\Delta_{h}^{m} f\right\|_{2, \gamma}^{2}=\sum_{k=0}^{\infty}\left[1-(1-h)^{k}\right]^{2 m}\left|c_{k}(f)\right|^{2}, \quad h \in(0,1),
$$

which afford to obtain (6) because of (5). Lemma is proved. We must note that the last equation was stated in [9, pp.1001] without proof.

In work [9] it was proved that for any arbitrary function $f \in L_{2, \gamma}$ for each $t \in(0,1)$ is hold an estimate

$$
E_{n-1}(f)_{2, \gamma} \leq\left[1-(1-t)^{n}\right]^{-m} \Omega_{m}(f ; t)_{2, \gamma}, m, n \in \mathbb{N},
$$

and for each fixed $n$ the constant in the right side of inequality (7) cannot be reduced. Indeed, on one hand for any function $f \in L_{2, \gamma}$ we find:

$$
\sup _{f \in L_{2, \gamma}} \frac{E_{n-1}(f)_{2, \gamma}}{\Omega_{m}(f ; t)_{2, \gamma}} \leq\left[1-(1-t)^{n}\right]^{-m} \text {. }
$$


On the other hand, as follows from (2) for the function $f_{0}(z)=\varphi_{n}(z)$, where $\varphi_{n}(z)$ is the $n$-th term of orthogonal system $\left\{\varphi_{k}(z)\right\}_{k=0}^{\infty}$, we hold $E_{n-1}\left(f_{0}\right)_{2, \gamma}=\left|c_{n}\left(f_{0}\right)\right|=1$. For the same function from (6) follows that

$$
\Omega_{m}\left(f_{0} ; t\right)_{2, \gamma}=\left[1-(1-t)^{n}\right]^{m}
$$

We hold

$$
\sup _{f \in L_{2, \gamma}} \frac{E_{n-1}(f)_{2, \gamma}}{\Omega_{m}(f ; t)_{2, \gamma}} \geq \frac{E_{n-1}\left(f_{0}\right)_{2, \gamma}}{\Omega_{m}\left(f_{0} ; t\right)_{2, \gamma}}=\left[1-(1-t)^{n}\right]^{-m} .
$$

Thus comparing the inequalities (9) and (10), we obtain

$$
\sup _{f \in L_{2, \gamma}} \frac{E_{n-1}(f)_{2, \gamma}}{\Omega_{m}(f ; t)_{2, \gamma}}=\frac{1}{\left(1-(1-t)^{n}\right)^{m}}, \quad 0<t<1 .
$$

When in (11) $t=1 / n$, we have

$$
\sup _{f \in L_{2, \gamma}} \frac{E_{n-1}(f)_{2, \gamma}}{\Omega_{m}(f ; 1 / n)_{2, \gamma}}=\left(1-\left(1-\frac{1}{n}\right)^{n}\right)^{-m}
$$

and this yields the equation

$$
\sup _{n \in \mathbb{N}} \sup _{f \in L_{2, \gamma}} \frac{E_{n-1}(f)_{2, \gamma}}{\Omega_{m}(f ; 1 / n)_{2, \gamma}}=\left(1-e^{-1}\right)^{-m}
$$

\section{Main Results}

Further and everywhere by weighted function in segment $[0, h]$ we shall understand a nonnegative measurable and summable in $[0, h]$ function $q(t)$ that is not equal to zero.

The following theorem is valid.

Theorem 1. Let $m, n \in \mathbb{N}, 0<p \leq 2,0<h<1, q-$ weighted function in $(0, h)$. Then is valid an equation

$$
\sup _{f \in L_{2, \gamma}} \frac{E_{n-1}(f)_{2, \gamma}}{\left(\int_{0}^{h} \Omega_{m}^{p}(f, t)_{2, \gamma} q(t) d t\right)^{\frac{1}{p}}}=\frac{1}{\left(\int_{0}^{h}\left(1-(1-t)^{n}\right)^{m p} q(t) d t\right)^{\frac{1}{p}}} .
$$

Proof. Using the following simplified form of Minkowsky's inequality [14, p.104]:

$$
\left(\int_{0}^{h}\left(\sum_{k=n}^{\infty}\left|\tilde{f}_{k}(t)\right|^{2}\right)^{\frac{p}{2}} d t\right)^{\frac{1}{p}} \geq\left(\sum_{k=n}^{\infty}\left(\int_{0}^{h}\left|\tilde{f}_{k}(t)\right|^{p} d t\right)^{\frac{2}{p}}\right)^{\frac{1}{2}}
$$

which is hold for any $0<p \leq 2$ and $h \in \mathbb{R}_{+}$. Taking $\tilde{f}_{k}=f_{k} q^{1 / p}$ in (13) we find

$$
\left(\int_{0}^{h}\left(\sum_{k=n}^{\infty}\left|f_{k}(t)\right|^{2}\right)^{\frac{p}{2}} q(t) d t\right)^{\frac{1}{p}} \geq\left(\sum_{k=n}^{\infty}\left(\int_{0}^{h}\left|f_{k}(t)\right|^{p} q(t) d t\right)^{\frac{2}{p}}\right)^{\frac{1}{2}} .
$$

By inequality (14), and equalities (6), (2) considering the obvious relation

$$
\inf _{k \geq n} \int_{0}^{h}\left(1-(1-t)^{k}\right)^{m p} q(t) d t=\int_{0}^{h}\left(1-(1-t)^{n}\right)^{m p} q(t) d t,
$$


we obtain

$$
\begin{gathered}
\left\{\int_{0}^{h} \Omega_{m}^{p}(f, t)_{2, \gamma} q(t) d t\right\}^{1 / p}=\left\{\int_{0}^{h}\left(\Omega_{m}^{2}(f, t)_{2, \gamma}\right)^{p / 2} q(t) d t\right\}^{1 / p} \geq \\
\geq\left\{\int_{0}^{h}\left(\sum_{k=n}^{\infty}\left(1-(1-t)^{k}\right)^{2 m}\left|c_{k}(f)\right|^{2}\right)^{p / 2} q(t) d t\right\}^{1 / p} \geq \\
\geq\left\{\sum_{k=n}^{\infty}\left|c_{k}(f)\right|^{2}\left(\int_{0}^{h}\left(1-(1-t)^{k}\right)^{m p} q(t) d t\right)^{2 / p}\right\}^{1 / 2} \geq \\
\geq\left(\int_{0}^{h}\left(1-(1-t)^{n}\right)^{m p} q(t) d t\right)^{1 / p} \cdot\left\{\sum_{k=n}^{\infty}\left|c_{k}(f)\right|^{2}\right\}^{1 / 2}= \\
=\left(\int_{0}^{h}\left(1-(1-t)^{n}\right)^{m p} q(t) d t\right)^{\frac{1}{p}} \cdot E_{n-1}(f)_{2, \gamma} .
\end{gathered}
$$

From (15) we obtain the above estimate in the right side of equation (12):

$$
\sup _{f \in L_{2, \gamma}} \frac{E_{n-1}(f)_{2, \gamma}}{\left(\int_{0}^{h} \Omega_{m}^{p}(f, t)_{2, \gamma} q(t) d t\right)^{\frac{1}{p}}} \leq \frac{1}{\left(\int_{0}^{h}\left(1-(1-t)^{n}\right)^{m p} q(t) d t\right)^{\frac{1}{p}}}
$$

For obtaining the bellow estimate of the same magnitude we assume that $f_{0}(z):=\varphi_{n}(z) \in L_{2, \gamma}$. Since for this function

$$
E_{n-1}\left(f_{0}\right)_{2, \gamma}=1, \quad \Omega_{m}\left(f_{0}, t\right)_{2, \gamma}=\left(1-(1-t)^{n}\right)^{m}, 0<t<1,
$$

therefore we hold

$$
\int_{0}^{h} \Omega_{m}^{p}\left(f_{0}, t\right)_{2, \gamma} q(t) d t=\int_{0}^{h}\left(1-(1-t)^{n}\right)^{m p} q(t) d t
$$

Consequently

$$
\begin{gathered}
\sup _{f \in L_{2, \gamma}} \frac{E_{n-1}(f)_{2, \gamma}}{\left(\int_{0}^{h} \Omega_{m}^{p}(f, t)_{2, \gamma} q(t) d t\right)^{1 / p}} \geq \frac{E_{n-1}\left(f_{0}\right)_{2, \gamma}}{\left(\int_{0}^{h} \Omega_{m}^{p}\left(f_{0}, t\right)_{2, \gamma} q(t) d t\right)^{1 / p}}= \\
=\frac{1}{\left(\int_{0}^{h}\left(1-(1-t)^{n}\right)^{m p} q(t) d t\right)^{\frac{1}{p}}} .
\end{gathered}
$$

Comparing the above estimate (16) and below estimate (17) we obtain an equation (12). This completes the proof of Theorem 1 .

The proved Theorem 1 implies the following corollaries.

Corollary 1. Let $m, n \in \mathbb{N}, p=1 / m, h \in(0,1), q$-weighted function in $(0, h)$. Then is valid an equation

$$
\sup _{f \in L_{2, \gamma}} \frac{E_{n-1}(f)_{2, \gamma}}{\left(\int_{0}^{h} \Omega_{m}^{1 / m}(f, t)_{2, \gamma} q(t) d t\right)^{m}}=\left(\int_{0}^{h}\left[1-(1-t)^{n}\right] q(t) d t\right)^{-m}
$$


From (18) in particularly when $q(t) \equiv 1$ follows an equation

$$
\sup _{f \in L_{2, \gamma}} \frac{E_{n-1}(f)_{2, \gamma}}{\left((n+1) \int_{0}^{h} \Omega_{m}^{\frac{1}{m}}(f, t)_{2, \gamma} d t\right)^{m}}=\frac{1}{\left\{(n+1) h-1+(1-h)^{n+1}\right\}^{m}} .
$$

Considering in (19) for example $h=1 /(n+1)$ we obtain

$$
\sup _{f \in L_{2, \gamma}} \frac{E_{n-1}(f)_{2, \gamma}}{\left((n+1) \int_{0}^{\frac{1}{n+1}} \Omega_{m}^{\frac{1}{m}}(f, t)_{2, \gamma} d t\right)^{m}}=\left(1-\frac{1}{n+1}\right)^{-m(n+1)}
$$

from which follows an extremal equality

$$
\sup _{n \in \mathbb{N} f \in L_{2, \gamma}} \frac{E_{n-1}(f)_{2, \gamma}}{\left((n+1) \int_{0}^{1 /(n+1)} \Omega_{m}^{1 / m}(f, t)_{2, \gamma} d t\right)^{m}}=e^{m} .
$$

Corollary 2. Let all condition of Theorem 1 be satisfied and $q(t)=n(1-t)^{n-1}, n \in \mathbb{N}$. Then for any $h \in(0,1)$ is valid an equation

$$
\sup _{f \in L_{2, \gamma}} \frac{E_{n-1}(f)_{2, \gamma}}{\left(n \int_{0}^{h} \Omega_{m}^{p}(f, t)_{2, \gamma}(1-t)^{n-1} d t\right)^{\frac{1}{p}}}=\left\{\frac{m p+1}{\left[1-(1-h)^{n}\right]^{m p+1}}\right\}^{\frac{1}{p}} .
$$

From (21) in particularly when $h=1 / n, n \in \mathbb{N}$ we obtain

$$
\sup _{n \in \mathbb{N} f \in L_{2, \gamma}} \frac{E_{n-1}(f)_{2, \gamma}}{\left(n \int_{0}^{\frac{1}{n}} \Omega_{m}^{p}(f, t)_{2, \gamma}(1-t)^{n-1} d t\right)^{\frac{1}{p}}}=\frac{(m p+1)^{\frac{1}{p}}}{\left(1-e^{-1}\right)^{m+\frac{1}{p}}} .
$$

In its turn from (22) when $p=1 / m, m \in \mathbb{N}$ follows an equation

$$
\sup _{n \in \mathbb{N} f \in L_{2, \gamma}} \frac{E_{n-1}(f)_{2, \gamma}}{\left(n \int_{0}^{1 / n} \Omega_{m}^{1 / m}(f, t)_{2, \gamma}(1-t)^{n-1} d t\right)^{m}}=2^{m}\left(\frac{e}{e-1}\right)^{2 m} .
$$

\section{The Exact Values of $n$-Widths for some Class of Functions.}

First, we recall the notions and definitions required for formulation of further results. Let $S$ be unit ball in space $L_{2, \gamma}, \Lambda_{n} \subset L_{2, \gamma}$ an $n$-dimensional subspace, $\Lambda^{n} \subset L_{2, \gamma}$ a subspace of codimension $n$, $\mathcal{L}: L_{2, \gamma} \rightarrow \Lambda_{n}$ a continuous linear operator, $\mathcal{L}^{\perp}: L_{2, \gamma} \rightarrow \Lambda_{n}$ a continuous linear projection operator, and $\mathfrak{M}$ be the convex centrally symmetric subset of $L_{2, \gamma}$. The quantities

$$
\begin{gathered}
b_{n}\left(, L_{2, \gamma}\right)=\sup \left\{\sup \left\{\varepsilon>0 ; \varepsilon S \cap \Lambda_{n+1} \subset\right\}: \Lambda_{n+1} \subset L_{2, \gamma}\right\}, \\
d_{n}\left(, L_{2, \gamma}\right)=\inf \left\{\sup \left\{\inf \left\{\|f-g\|_{2}: g \in \Lambda_{n}\right\}: f \in\right\}: \Lambda_{n} \subset L_{2, \gamma}\right\}, \\
\delta_{n}\left(, L_{2, \gamma}\right)=\inf \left\{\inf \left\{\sup \left\{\|f-\mathcal{L} f\|_{2}: f \in\right\}: \mathcal{L} L_{2, \gamma} \subset \Lambda_{n}\right\}: \Lambda_{n} \subset L_{2, \gamma}\right\}, \\
d^{n}\left(, L_{2, \gamma}\right)=\inf \left\{\sup \left\{\|f\|_{2, \gamma}: f \in \cap \Lambda^{n}\right\}: \Lambda^{n} \subset L_{2, \gamma}\right\}, \\
\Pi_{n}\left(, L_{2, \gamma}\right)=\inf \left\{\inf \left\{\sup \left\{\left\|f-\mathcal{L}^{\perp} f\right\|_{2}: f \in\right\}: \mathcal{L}^{\perp} L_{2, \gamma} \subset \Lambda_{n}\right\}: \Lambda_{n} \subset L_{2, \gamma}\right\}
\end{gathered}
$$


are called the Bernshtein, Kolmogorov, linear, Gelfand and projection n-widths of subset $\mathfrak{M}$ in $L_{2, \gamma}$. In $[14,15]$ it is known that for the above $n$-widths are monotony and in Hilbert space $L_{2, \gamma}$ there is hold the following relations

$$
b_{n}\left(\mathfrak{M}, L_{2, \gamma}\right) \leq d^{n}\left(\mathfrak{M}, L_{2, \gamma}\right) \leq d_{n}\left(\mathfrak{M}, L_{2, \gamma}\right)=\delta_{n}\left(\mathfrak{M}, L_{2, \gamma}\right)=\Pi_{n}\left(\mathfrak{M}, L_{2, \gamma}\right) .
$$

We introduce the following class of functions which lead from (8) and Theorem 1. Let $h \in(0,1)$, $m \in \mathbb{N}$. By $W_{2, \gamma, m}(\Phi, h)$ we denote class of function $f \in L_{2, \gamma}$ where the generalized module of continuity (6) satisfies the condition

$$
\Omega_{m}(f, h)_{2, \gamma} \leq \Phi(h),
$$

where $\Phi$ is nonnegative and monotony increasing function in $[0,+\infty)$.

By $W_{p} L_{2, \gamma}\left(\Omega_{m} ; q, h\right)$, where $m \in \mathbb{N}, h \in(0,1)$ and $0<p \leq 2$, we denote class of functions $f \in L_{2, \gamma}$, satisfy the condition

$$
\left(\int_{0}^{h} \Omega_{m}^{p}(f ; t)_{2, \gamma} q(t) d t\right)^{1 / p} \leq 1 .
$$

Theorem 2. For any $n, m \in \mathbb{N}$ and $h \in(0,1)$ is valid an equation

$$
\lambda_{n}\left(W_{2, \gamma, m}(\Phi, h), L_{2, \gamma}\right)=E_{n-1}\left(W_{2, \gamma, m}(\Phi, h)\right)=\left[1-(1-h)^{n}\right]^{-m} \Phi(h),
$$

where $\lambda_{n}(\cdot)$ is any of $n$-widths $b_{n}(\cdot), d_{n}(\cdot), d^{n}(\cdot), \delta_{n}(\cdot), \Pi_{n}(\cdot)$ and

$$
E_{n-1}\left(W_{2, \gamma, m}(\Phi, h)\right)=\sup \left\{E_{n-1}(f)_{2, \gamma}: f \in W_{2, \gamma, m}(\Phi, h)\right\} .
$$

Proof. The above estimate of all considered $n$-widths of class $W_{2, \gamma, m}(\Phi, h)$ will follows from (8), as

$$
\begin{gathered}
E_{n-1}\left(W_{2, \gamma, m}(\Phi)\right)=\sup _{f \in W_{2, \gamma, m}(\Phi)} E_{n-1}(f)_{2, \gamma} \leq \\
\leq \sup _{f \in W_{2, \gamma, m}(\Phi)}\left\{\left[1-(1-h)^{n}\right]^{-m} \Omega_{m}(f, h)_{2, \gamma}\right\} \leq\left[1-(1-h)^{n}\right]^{-m} \Phi(h) .
\end{gathered}
$$

Hence, according to relation (23) for all listed $n$-widths we will obtain the above estimate

$$
\lambda_{n}\left(W_{2, \gamma, m}(\Phi, h)\right) \leq\left[1-(1-h)^{n}\right]^{-m} \Phi(h) .
$$

In order to find the below estimate of all $n$-widths of the right side of $(26)$ in $(n+1)$-dimension subspace of generalized polynomials

$$
\mathcal{P}_{n+1}=\left\{p_{n+1}(z): p_{n+1}(z)=\sum_{k=0}^{n} a_{k} \varphi_{k}(z)\right\},
$$

we will consider the ball

$$
S_{n+1}:=\left\{p_{n+1}(z) \in \mathcal{P}_{n+1}:\left\|p_{n+1}\right\|_{2, \gamma} \leq\left[1-(1-h)^{n}\right]^{-m} \Phi(h)\right\},
$$

and show that the ball $S_{n+1} \subset W_{2, \gamma, m}(\Phi)$. Indeed, for arbitrary $p_{n+1}(z) \in S_{n+1}$, according to (6) we hold:

$$
\begin{gathered}
\Omega_{m}^{2}\left(p_{n+1} ; h\right)_{2, \gamma}=\sum_{k=0}^{n}\left[1-(1-h)^{k}\right]^{2 m}\left|a_{k}\left(p_{n+1}\right)\right|^{2} \leq \\
\leq\left[1-(1-h)^{n}\right]^{2 m} \sum_{k=0}^{n}\left|a_{k}\left(p_{n+1}\right)\right|^{2}=\left[1-(1-h)^{n}\right]^{2 m}\left\|p_{n+1}\right\|_{2, \gamma}^{2} \leq \\
\leq\left[1-(1-h)^{n}\right]^{2 m} \cdot\left[1-(1-h)^{n}\right]^{-2 m} \Phi^{2}(h)=\Phi^{2}(h) .
\end{gathered}
$$


Thus, we proved that for arbitrary $p_{n+1} \subset S_{n+1}$ is hold an inequality $\Omega_{m}\left(p_{n+1}, h\right)_{2, \gamma} \leq \Phi(h)$, and this means that $S_{n+1} \subset W_{2, \gamma, m}(\Phi, h)$. Then, according to the definition of Bernshtein $n$-width and relation (23) we write

$$
\begin{gathered}
\lambda_{n}\left(W_{2, \gamma, m}(\Phi, h), L_{2, \gamma}\right) \geq b_{n}\left(W_{2, \gamma, m}(\Phi, h), L_{2, \gamma}\right) \geq \\
\geq b_{n}\left(S_{n+1}, L_{2, \gamma}\right) \geq\left[1-(1-h)^{n}\right]^{-m} \Phi(h) .
\end{gathered}
$$

The proof of Theorem 2 will be followed by comparison of above estimate (26) and below estimate (27).

We note that the statement of Theorem 2 for Kolmogorov $n$-width had been proved before in [9].

Theorem 2 implies the following statement.

Corollary 3. In theorem 2 when $h=1 / n, n \in \mathbb{N}$ there is hold an asymptotic equation

$$
\lambda_{n}\left(W_{2, \gamma, m}(\Phi, h), L_{2, \gamma}\right)=\left[1-\left(1-\frac{1}{n}\right)^{n}\right]^{-m} \Phi\left(\frac{1}{n}\right) \sim\left(1-e^{-1}\right)^{-m} \Phi\left(\frac{1}{n}\right) .
$$

Theorem 3. Let $m \in \mathbb{N}, 0<p \leq 2, h \in(0,1), q \geq 0$ is a weighted function in $(0, h)$. Then for any $n \in \mathbb{N}$ are valid the equations

$$
\begin{gathered}
\lambda_{n}\left(W_{p} L_{2, \gamma}\left(\Omega_{m} ; q, h\right), L_{2, \gamma}\right)=E_{n-1}\left(W_{p} L_{2, \gamma}\left(\Omega_{m} ; q, h\right)\right)= \\
=\left(\int_{0}^{h}\left(1-(1-t)^{n}\right)^{m p} q(t) d t\right)^{-\frac{1}{p}},
\end{gathered}
$$

where $\lambda_{n}(\cdot)$ any of listed above $n$-widths.

Proof. The above estimate for all aforementioned $n$-widths we will obtain from (16), (23) and by the definition of class of function $W_{p} L_{2, \gamma}\left(\Omega_{m} ; q, h\right)$

$$
\begin{aligned}
& \lambda_{n}\left(W_{p} L_{2, \gamma}\left(\Omega_{m} ; q, h\right), L_{2, \gamma}\right) \leq d_{n}\left(W_{p} L_{2, \gamma}\left(\Omega_{m} ; q, h\right), L_{2, \gamma}\right) \leq \\
\leq & E_{n-1}\left(W_{p} L_{2, \gamma}\left(\Omega_{m} ; q, h\right)\right) \leq\left(\int_{0}^{h}\left(1-(1-t)^{n}\right)^{m p} q(t) d t\right)^{-\frac{1}{p}} .
\end{aligned}
$$

For obtaining an estimate below on set $\mathcal{P}_{n} \cap L_{2, \gamma}$ we consider the ball

$$
\sigma_{n+1}=\left\{p_{n+1} \subset \mathcal{P}_{n+1}:\left\|p_{n+1}\right\|_{2, \gamma} \leq\left(\int_{0}^{h}\left(1-(1-t)^{n}\right)^{m p} q(t) d t\right)^{-1 / p}\right\}
$$

and we prove an inclusion of $\sigma_{n+1} \subset W_{p} L_{2, \gamma}\left(\Omega_{m} ; q, h\right)$.

For arbitrary polynom $p_{n+1} \subset \sigma_{n+1}$ according to (6) we write

$$
\begin{gathered}
\Omega_{m}^{2}\left(p_{n+1} ; t\right)_{2, \gamma}=\sum_{k=0}^{n}\left(1-(1-t)^{k}\right)^{2 m}\left|c_{k}\left(p_{n+1}\right)\right|^{2} \leq \\
\leq\left(1-(1-t)^{n}\right)^{2 m} \sum_{k=0}^{n}\left|c_{k}\left(p_{n+1}\right)\right|^{2}=\left(1-(1-t)^{n}\right)^{2 m} \cdot\left\|p_{n+1}\right\|_{2, \gamma}^{2}
\end{gathered}
$$

or as the same

$$
\Omega_{m}\left(p_{n+1} ; t\right)_{2, \gamma} \leq\left(1-(1-t)^{n}\right)^{m} \cdot\left\|p_{n+1}\right\|_{2, \gamma}
$$


Raising both side of inequality (30) to the power $p$, multiplying them by weighted function $q$ and integrating both side with respect $t$ in the limits from $t=0$ to $t=h$ we have

$$
\begin{aligned}
& \int_{0}^{h} \Omega_{m}^{p}\left(p_{n+1} ; t\right)_{2, \gamma} q(t) d t \leq\left\|p_{n+1}\right\|_{2, \gamma}^{p} \cdot \int_{0}^{h}\left(1-(1-t)^{n}\right)^{m p} q(t) d t \leq \\
& \leq\left(\int_{0}^{h}\left(1-(1-t)^{n}\right)^{m p} q(t) d t\right)^{-1} \cdot \int_{0}^{h}\left(1-(1-t)^{n}\right)^{m p} q(t) d t=1,
\end{aligned}
$$

and, thus the inclusion of $\sigma_{n+1} \subset W_{p} L_{2, \gamma}\left(\Omega_{m} ; q, h\right)$ is proved. By the definition of Bernshtein $n$-width and relation (23) between the $n$-widths we hold

$$
\begin{gathered}
\lambda_{n}\left(W_{p} L_{2, \gamma}\left(\Omega_{m} ; q, h\right), L_{2, \gamma}\right) \geq b_{n}\left(W_{p} L_{2, \gamma}\left(\Omega_{m} ; q, h\right), L_{2, \gamma}\right) \geq \\
\geq b_{n}\left(\sigma_{n+1}, L_{2, \gamma}\right) \geq\left(\int_{0}^{h}\left(1-(1-t)^{n}\right)^{m p} q(t) d t\right)^{-\frac{1}{p}} .
\end{gathered}
$$

We obtain the required equation (28) by comparing the above (29) and below (31) estimates. Theorem 3 is proved.

\section{References}

[1] M.Sh. Shabozov, O.Sh. Shabozov, Best approximation and the value of the widths of some classes of functions in the Bergman space $B_{p}, 1 \leq p \leq \infty$, Dokl. Akad. Nauk. 410(4) (2006) 461-464.

[2] M.Sh. Shabozov, O.Sh. Shabozov, On the best approximation of some classes of analytic functions in the weighted Bergman space $B_{2, \gamma}$, Dokl. Akad. Nauk. 412(4) (2007) 466-469.

[3] S.B. Vakarchuk, M.Sh. Shabozov, The widths of classes of analytic functions in a disc, Mat. Sb. 201(8) (2010) 3-22 (in Russian). Translated in: Sbornik: Mathematics. 201(8) (2010) 1091.

[4] M.Sh. Shabozov, M.S. Saidusaynov, On the values of widths and the best linear method of approximation in the weighted Bergman space, Proceedings of the TSU. Natural Science. 3 (2014) 40-57. (in Russian)

[5] M.S. Saidusaynov, On the values of widths and the best linear method of approximation in the weighted Bergman space, Proceedings of the TSU. Natural Science. 3 (2015) 91-104. (in Russian)

[6] M.S. Saidusaynov, On the best linear method of approximation of some classes analytic functions in the weighted Bergman space, Chebyshevskii Sbornik. 1 (2016) 246-260. (in Russian)

[7] M.R. Langarshoev, On the best linear methods of approximation and the exact values of widths for some classes of analytic functions in the weighted Bergman space, Ukr. Mat. Zh. 67(10) (2015) 1366-1379 (in Russian). Translated in: Ukrainian Mathematical Journal. 67(10) (2016) 1537-1551.

[8] V.I. Smirnov, N.A. Lebedev, Constructive functions theory of complex variables, Nauka, Moscow - Leningrad, USSR, 1964. (in Russian)

[9] V.A. Abilov, F.V. Abilova, M.K. Kerimov, Sharp estimates for the rate of convergence of Fourier series of functions of a complex variable in the space $L_{2}(D, p(z))$, Zh. Vychisl. Mat. Fiz. 50(6) (2010) 999-1004 (in Russian). Translated in: Computational Mathematics and Mathematical Physics. 50(6) (2010) 946-950. 
[10] V.A. Abilov, F.V. Abilova, M.K. Kerimov, Sharp estimates for the convergence rate of Fourier series in terms of orthogonal polynomials in $L_{2}((a, b), p(x)), \mathrm{Zh}$. Vychisl. Mat. Fiz. 49(6) (2009) 966-980 (in Russian). Translated in: Computational Mathematics and Mathematical Physics. 49(6) (2009) 927-941

[11] S.B. Vakarchuk, A.V. Shvachko, On the best approximation in the mean by algebraic polynomials with weight and the exact values of widths for the classes of functions, Ukr. Mat. Zh. 65(12) (2013) 1604-1621 (in Russian). Translated in: Ukrainian Mathematical Journal. 65(12) (2014) 1774-1792.

[12] S.B. Vakarchuk, Mean approximation of functions on the real axis by algebraic polynomials with Chebyshev-Hermite weight and widths of function class, Mat. Zametki. 95(5) (2014) 666-684 (in Russian). Translated in: Mathematical Notes. 95(5) (2014) 599-614.

[13] M.Sh. Shabozov, K. Tukhliev, Jackson-Stechkin type inequalities with generalized moduli of continuity and widths of some classes of functions, Trudy Inst. Mat. i Mekh. UrO RAN. 21(4) (2015) 292-308. (in Russian)

[14] A. Pinkus, n-Widths in Approximation Theory, Springer-Verlag, Berlin, Heidelberg, New York, Tokyo, 1985.

[15] V.M. Tikhomirov, Some problems of theory of approximation, MSU, Moscow, USSR, 1976. (in Russian) 